\title{
Murine models of Omenn syndrome
}

\author{
Serre-Yu Wong and David B. Roth
}

\author{
Program in Molecular Pathogenesis, Skirball Institute of Biomolecular Medicine, and Department of Pathology, \\ New York University School of Medicine, New York, New York, USA.
}

\begin{abstract}
In the 40 years since Harvard medical student Gilbert Omenn first described a rare, inherited disorder producing a paradoxical combination of immunodeficiency and immune dysregulation, the pathogenesis of Omenn syndrome (OS) has remained mysterious. In separate studies reported in this issue of the JCI, two mouse models bearing mutations in the V(D)J recombinase analogous to those causing human OS have been shown to recapitulate the disease and provide insight into the genesis of immunodeficiency combined with autoimmunity and atopy in OS and other disease settings (see the related articles beginning on pages 1260 and 1270).
\end{abstract}

\section{A perplexing combination of immunologic symptoms}

Omenn syndrome (OS) is differentiated from SCID by the additional features of autoimmunity and atopy, which signify substantial immune dysregulation (1-4). Although the clinical picture of OS is variable, patients typically present in infancy with erythroderma, alopecia, hepatosplenomegaly, lymphadenopathy, chronic diarrhea, failure to thrive, and recurrent infections. Peripheral blood analyses reveal eosinophilia, severely decreased B cell counts, and hypogammaglobulinemia, but markedly elevated IgE levels. Although T cell counts are normal to elevated, $\mathrm{T}$ cells display a highly restricted, oligoclonal TCR repertoire (ref. 3 and references therein). When left untreated, OS is fatal, usually as a result of infections; the available therapies, bone marrow and cord blood stem cell transplantation, are variably successful $(2,4)$.

OS can be caused by any of several genetic defects that impair lymphocyte development. Mutations affecting (a) thymic development (resulting in atypical DiGeorge syndrome); (b) cytokine receptors required for $\mathrm{T}$ cell development (e.g., IL-7 receptor deficiency); or (c) mitochondrial RNA processing (e.g., the $R N A$ component of mitochondrial RNA processing endoribonuclease gene, RMRP) have been reported $(3,5)$. The best-characterized defects, however, are homozygous or compound heterozygous mutations

Nonstandard abbreviations used: AIRE, autoimmune regulator; OS, Omenn syndrome.

Conflict of interest: The authors have declared that no conflict of interest exists.

Citation for this article: J. Clin. Invest. 117:1213-1216 (2007). doi:10.1172/JCI32214. in either RAG1 or RAG2, which together encode the protein complex that catalyzes $\mathrm{V}(\mathrm{D}) \mathrm{J}$ recombination (6). This process, whereby DNA rearrangements at the TCR and Ig gene loci create a diverse repertoire of receptors, is required for lymphocyte development. The RAG mutations seen in OS are characteristically hypomorphic, partially disabling the activity of the $V(D) J$ recombinase (hypomorphic mutations in Artemis, a DNA repair gene crucial for $\mathrm{V}(\mathrm{D}) \mathrm{J}$ recombination, can also cause OS; ref. 3). In contrast to severe, inactivating mutations in the RAG genes that lead to SCID, a hypomorphic $V(D) J$ recombinase that is able to generate only a few productive antigen receptor gene rearrangements accounts for the immunodeficiency and oligoclonal repertoire in OS $(7,8)$.

A compelling explanation for the connection between a severely constrained TCR repertoire and the atopic/autoimmune features of OS has proven elusive. Pathogenesis is likely multifactorial. The involvement of modifying genes and epigenetic influences have been suggested (8), although no supporting evidence has yet emerged. Exposure to foreign antigens could also play a role: antigenic stimulation from recurrent infections could facilitate expansion of lymphocyte clones, including some with self reactivity (7-9). This idea is supported by the observation that a patient with $\mathrm{T}^{-} \mathrm{B}^{-}$SCID progressed to OS following a parainfluenza virus infection (10). Iatrogenic components, including prolonged courses of antibiotic and immunosuppressive therapy, could also influence the development or phenotypic expression of OS (8). One possible scenario is that these treatments alter the commensal microbial flora, thought to be important for immunologic tolerance by affecting the development of a functional Treg compartment (11). A peripheral imbalance of Tregs has also been mentioned as a possible cause of the loss of tolerance in OS $(2,3)$. Most recently, diminished expression of autoimmune regulator (AIRE) was found in the thymi of patients with OS, suggesting a mechanism for the impairment of negative selection $(2,12)$. The lack of an animal model for OS has hindered analysis of the contributions by these factors to the disease. Now, in this issue of the JCI, two groups have characterized two mouse models that recapitulate key features of human OS.

\section{Two mouse models of OS}

In the first report, Marella et al. (13) created knockin mice bearing a Rag2 mutation (R229Q) identified in several OS patients, including those in families with histories of both OS and SCID $(8,9)$. B and T lymphocyte precursors were arrested in early developmental stages, consistent with a previous finding that a mutation in the same residue, R229A, severely reduces recombination activity in transfected cells (14). Sixty percent of the homozygous mice developed alopecia; a small percentage of the mice also developed severe erythroderma and a wasting syndrome, replicating important features of human OS. Regardless of severity of clinical manifestations, all Rag2 R229Q mutant mice displayed abnormal architecture of the thymus, lymph nodes, and spleen, consistent with known OS pathology, as well as restricted TCR repertoires, eosinophilia, and hypogammaglobulinemia; only some mice developed hyper-IgE syndrome. Notably, Marella et al. observed that Aire expression in thymi was low in mutant mice and that the proportion of Foxp $3^{+}$Tregs was diminished, offering important clues as to the defects in central and peripheral tolerance in OS (13).

Independently, Khiong and colleagues (15) discovered a spontaneous Rag1 mutation (R972Q), identical to a mutation found in an OS patient (8), that resulted in elevated memory-type $\mathrm{T}$ cell numbers in a C57BL/10 mouse. Development of lymphocyte precursors in homozygous Rag1 
A Normal

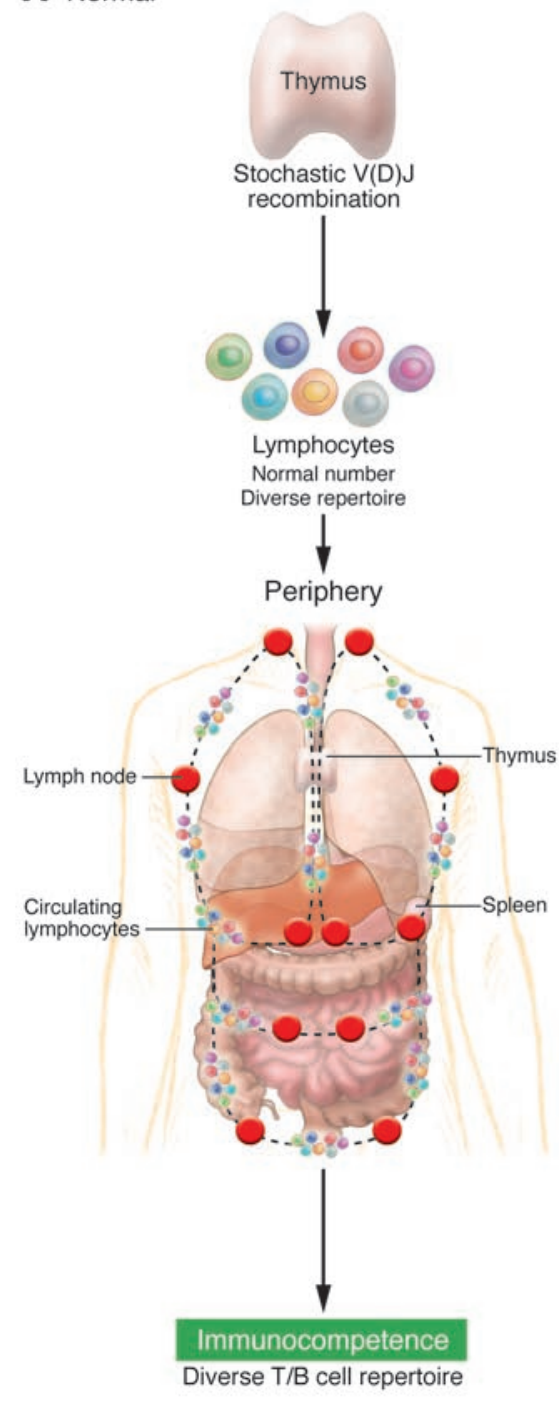

B Omenn syndrome

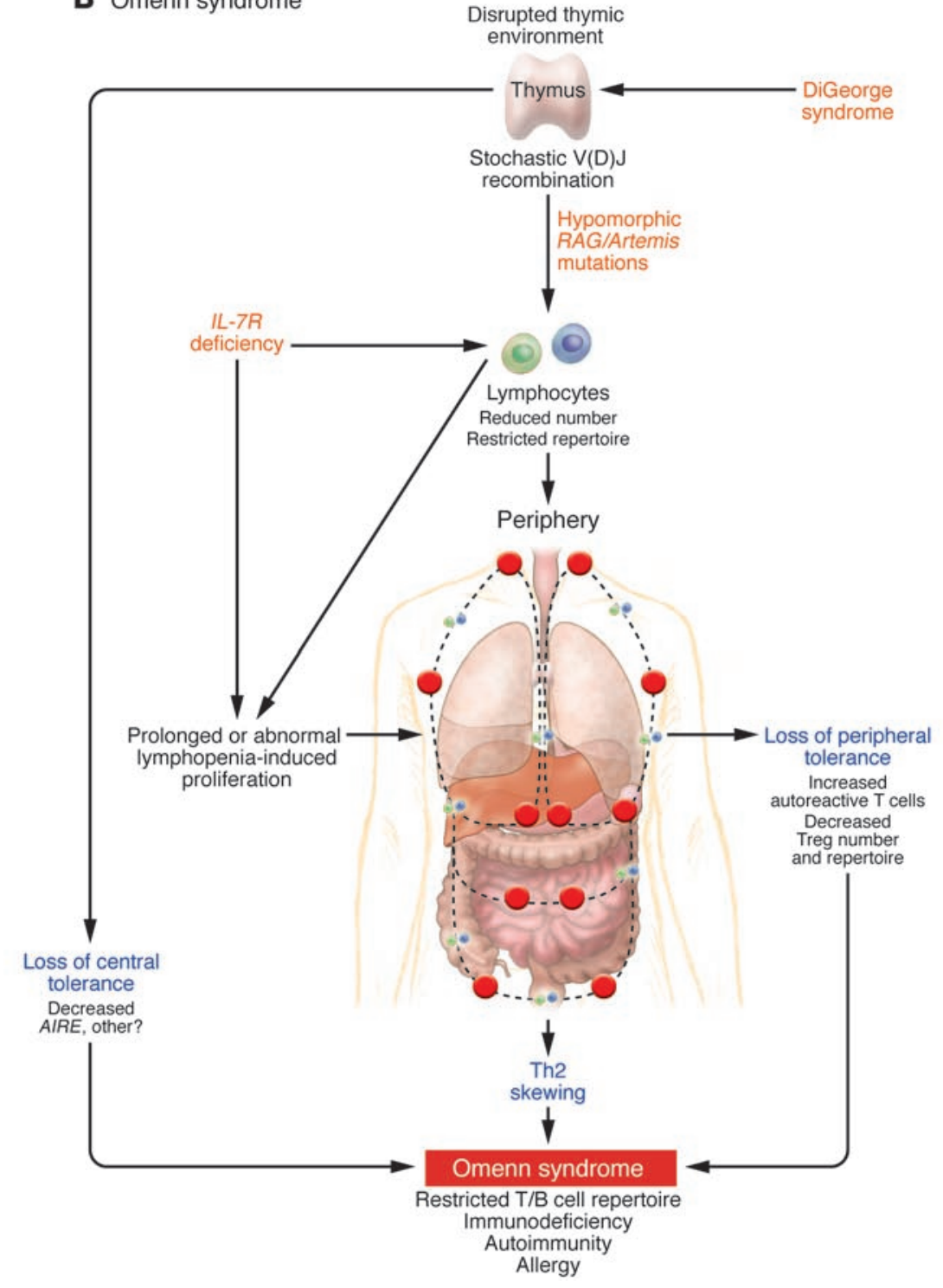

Figure 1

Schematic for the development of immunodeficiency and allergy/autoimmunity in OS. (A) Normally, V(D)J recombination is competent to produce a diverse repertoire of TCR molecules. In the thymus, normal selection processes are intact, and in the periphery, unimpeded lymphocyte development and handling of antigenic stimulation result in an immunocompetent individual. (B) In OS, genetic defects result in a restricted TCR repertoire and lymphopenia. A disrupted thymic environment, directly or indirectly caused by genetic defects, contributes to the loss of central tolerance. In the periphery, lymphopenia and IL-7 receptor $(I L-7 R)$ deficiency result in prolonged or abnormal homeostatic proliferation, which may result in Th2 skewing and loss of peripheral tolerance. In this issue of the $J C l$, Khiong et al. (15) implicate homeostatically proliferating $\mathrm{T}$ cells as a cause of Th2 skewing in a mutant mouse model of OS. Also in this issue, Marella et al. (13) show that in a mutant mouse model of OS, there is a decreased proportion of Foxp3 ${ }^{+}$Tregs in peripheral lymphoid organs. Other factors, including genetic, epigenetic, environmental, and iatrogenic factors, may influence disease development at any or all stages. The involvement of a severely restricted B cell repertoire remains unexplained in OS and is not depicted here.

R972Q mutant mice was arrested, consistent with the severe DNA cleavage defect in vitro of a similar mutant (R972A) (16). The authors noted pronounced skin redness (though without immune infiltrates), eosinophilia, elevated IgE levels, oligoclonal $\mathrm{T}$ cells, and pathology of thymus, lymph nodes, and spleen. At 25 weeks the mice developed hepatosplenomegaly. It is interesting that, unlike most OS patients, the mice developed elevated IgM, IgG2a, and IgG2b levels (15). Importantly, Khiong et al. address the mechanism underlying atopy in OS: the Rag1 R972Q mutant mice have increased secretion of Th2 cytokines IL-4 and IL-6, and the hyper-IgE phenotype is dependent on these cytokines and on $\mathrm{CD}^{+} \mathrm{T}$ cells (15). In addition, the lymphoid compartment of the Rag1 R972Q mutant mice is primed for lymphocyte expansion, as wild-type donor $\mathrm{CD}^{+}$and $\mathrm{CD}^{+} \mathrm{T}$ cells expanded in mutant, but not wild-type, mice (15). This implies that homeostatic proliferation, also known as lymphopenia- 
induced proliferation, is important for OS pathogenesis, a conclusion supported by the presence of surface activation markers on $\mathrm{T}$ cells in the mutant mice and in humans with OS $(2,3,13,15)$.

\section{A new view of OS pathogenesis}

We have integrated these findings into a comprehensive model for OS (Figure 1). Notably, both studies $(13,15)$ demonstrate that OS can develop in a controlled, specific pathogen-free environment; these observations suggest that variation in phenotypic expression of this disease is not solely due to environmental influences.

This model assumes conditions that diminish the generation of lymphocytes yet allow a few clones to be generated, resulting in lymphopenia and associated restricted $\mathrm{T}$ and $\mathrm{B}$ cell repertoire diversity (17). This is the case in OS patients - and mice - with hypomorphic RAG mutations and is consistent with other genetic causes of OS that critically limit the generation of lymphocytes. The stochastic nature of TCR and Ig rearrangements assures that no two individuals have the same antigen receptor repertoire. This influences the phenotypic expression of OS because the clones available for expansion in a given individual, combined with the individual's immunologic experience, should determine the incidence of autoreactivity and perhaps the number of Tregs. Such a model provides an explanation for the variability of clinical features expressed by OS patients, or mice, bearing the same mutant allele. It also predicts that the Treg repertoire is restricted in the OS mice, although this was not addressed by either study.

In this model, central tolerance is impaired by disruption of the thymic microenvironment, which is caused by deficient thymic development in atypical DiGeorge syndrome or by low throughput of thymocytes secondary to crippled $V(D) J$ recombination or $I L-7$ receptor deficiency. Altered thymic architecture and concomitant decreased Aire expression in the thymic medullary epithelium result in a failure of negative selection, allowing the survival of autoreactive $\mathrm{T}$ cell clones and a reduction of centrally derived Tregs (13).

Abnormal conditions in the periphery may also promote immune dysregulation in OS. The genetic mutations that underlie OS result in lymphopenia $(18,19)$, which activates compensatory proliferation of peripheral $\mathrm{T}$ cells. This in turn involves self-MHC interactions and production of cytokines (IL-7, IL-15, and IL-21) and, likely, Tregs (20,21). Indeed, Khiong et al. demonstrate that $\mathrm{T}$ cell expansion is favored in their mutant mice (15). It has been suggested that prolonged lymphopeniainduced homeostatic proliferation causes expansion of a few self-reactive clones with effector/memory-like phenotypes, as found in humans and mice with OS (18). A negative balance of Tregs, as found by Marella et al. (13), may further account for the loss of peripheral tolerance.

Regarding the allergic features of OS, experiments using the Rag1 mutant mice implicate proliferating $\mathrm{T}$ cells in the development of hyper-IgE secretion in OS (15). Others have suggested that homeostatic proliferation in OS generates Th2-skewed cells, and a restricted $T$ cell repertoire can cause Th2-skewed multiorgan symptoms in lymphopenic mice $(20,22)$. Intrinsic defects in B cells may also facilitate atopy in OS. Mutant RAG proteins might create a deficit in receptor editing, in which $I g$ gene rearrangements encoding autoreactive receptors are revised by peripheral $\mathrm{V}(\mathrm{D}) \mathrm{J}$ recombination, allowing the persistence of autoantibody-producing B cells (23). Increased antigenic stimulation caused by inefficient foreign antigen removal, elevated levels of IL-4, and increased cell cycling could give rise to Th2 skewing and IgE switching (ref. 20 and references therein). Finally, germinal centers in OS mice are dysmorphic and have few mature B cells, thereby creating an immature germinal center phenotype, which favors IgE production (24).

\section{Future directions}

Khiong et al. (15) and Marella et al. (13) establish their Rag mutant mice as valid murine models for OS. Future studies of these mice may clarify the source of the phenotypic variation in OS, distinguishing the contributions of environmental, genetic, and perhaps epigenetic factors. It may be useful to look for additional phenotypic abnormalities, such as the possible involvement of other organs and the fate of $\gamma \delta \mathrm{T}$ cells. Moreover, differences between OS presentations in humans and mice and between the two mouse models should be investigated, such as the Th1 skewing upon stimulation in the Rag2 R229Q mutant mice; the elevated IgM, IgG2a, and IgG2b in the Rag1 R972Q mutant mice; and the delayed disease onset in the OS mice (around 8 weeks of age - young adulthood for a mouse - as opposed to infancy in a human).
These murine models for OS constitute powerful tools with which to study the mechanisms underlying this complex and fascinating disease and may facilitate development of new therapeutic approaches. Finally, studies using these animals may illuminate the pathogenesis of other disorders characterized by combinations of immunodeficiency and autoimmunity: other primary immunodeficiencies, such as common variable immunodeficiency and Wiskott-Aldrich syndrome; various autoimmune diseases; and some cases of HIV infection $(2,18,21)$.

\section{Acknowledgments}

We thank Giulia Celli, Carlos E. Tadokoro, Vicky Brandt, Jennifer Posey, Rohit Chandwani, and the members of the Roth laboratory for their support and thoughtful critique of this commentary.

Address correspondence to: David B. Roth, Skirball Institute of Biomolecular Medicine, New York University School of Medicine, 540 First Avenue, Lab 2-10, New York, New York 10016, USA. Phone: (212) 263-0945; Fax: (212) 263-5711; E-mail: roth@saturn. med.nyu.edu.

1. Omenn, G.S. 1965. Familial reticuloendotheliosis with eosinophilia. N. Engl. J. Med. 273:427-432.

2. Notarangelo, L.D., Gambineri, E., and Badolato, R. 2006. Immunodeficiencies with autoimmune consequences. Adv. Immunol. 89:321-370.

3. Honig, M., and Schwarz, K. 2006. Omenn syndrome: a lack of tolerance on the background of deficient lymphocyte development and maturation. Curr. Opin. Rheumatol. 18:383-388.

4. Aleman, K., Noordzij, J.G., de Groot, R., van Dongen, J.J., and Hartwig, N.G. 2001. Reviewing Omenn syndrome. Eur. J. Pediatr. 160:718-725.

5. Roifman, C.M., Gu, Y., and Cohen, A. 2006. Mutations in the RNA component of RNase mitochondrial RNA processing might cause Omenn syndrome. J. Allergy Clin. Immunol. 117:897-903.

6. Sobacchi, C., Marrella, V., Rucci, F., Vezzoni, P., and Villa, A. 2006. RAG-dependent primary immunodeficiencies. Hum. Mutat. 27:1174-1184.

7. Villa, A., et al. 1998. Partial V(D)J recombination activity leads to Omenn syndrome. Cell. 93:885-896.

8. Villa, A., et al. 2001. V(D)J recombination defects in lymphocytes due to RAG mutations: severe immunodeficiency with a spectrum of clinical presentations. Blood. 97:81-88.

9. Corneo, B., et al. 2001. Identical mutations in RAG1 or RAG2 genes leading to defective $V(D) J$ recombinase activity can cause either T-B-severe combined immune deficiency or Omenn syndrome. Blood. 97:2772-2776.

10. Dalal, I., et al. 2005. Evolution of a T-B- SCID into an Omenn syndrome phenotype following parainfluenza 3 virus infection. Clin. Immunol. 115:70-73.

11. Ostman, S., Rask, C., Wold, A.E., Hultkrantz, S., and Telemo, E. 2006. Impaired regulatory T cell function in germ-free mice. Eur. J. Immunol. 36:2336-2346.

12. Cavadini, P., et al. 2005. AIRE deficiency in thymus 
of 2 patients with Omenn syndrome. J. Clin. Invest. 115:728-732. doi:10.1172/JCI200523087.

13. Marrella, V., et al. 2007. A hypomorphic R229Q Rag2 mouse mutant recapitulates human Omenn syndrome. J. Clin. Invest. 117:1260-1269. doi:10.1172/JCI30928.

14. Qiu, J.X., Kale, S.B., Yarnell Schultz, H., and Roth, D.B. 2001. Separation-of-function mutants reveal critical roles for RAG2 in both the cleavage and joining steps of V(D)J recombination. Mol. Cell. 7:77-87

15. Khiong, K., et al. 2007. Homeostatically proliferating $\mathrm{CD}^{+} \mathrm{T}$ cells are involved in the pathogenesis of an Omenn syndrome murine model. J. Clin. Invest. 117:1270-1281. doi:10.1172/JCI30513.

16. Lu, C.P., Sandoval, H., Brandt, V.L., Rice, P.A., and Roth, D.B. 2006. Amino acid residues in Rag1 crucial for DNA hairpin formation. Nat. Struct. Mol. Biol. 13:1010-1015

17. Min, B., Foucras, G., Meier-Schellersheim, M., and Paul, W.E. 2004. Spontaneous proliferation, a response of naive CD4 $\mathrm{T}$ cells determined by the diversity of the memory cell repertoire. Proc. Natl. Acad. Sci. U. S. A. 101:3874-3879.

18. Baccala, R., and Theofilopoulos, A.N. 2005. The new paradigm of T-cell homeostatic proliferationinduced autoimmunity. Trends Immunol. 26:5-8.

19. Curotto de Lafaille, M.A., Shen, S., Olivares-Villagomez, D., Camps-Ramirez, M., and Lafaille, J.J. 2005. Do regulatory T cells play a role in the control of homeostatic proliferation? Int. Rev. Immunol. 24:269-284.

20. Curotto de Lafaille, M.A., and Lafaille, J.J. 2004. The role of regulatory $\mathrm{T}$ cells in allergy. Springer Semin.
Immunopathol. 25:295-310.

21. Krupica, T., Jr., Fry, T.J., and Mackall, C.L. 2006. Autoimmunity during lymphopenia: a two-hit model. Clin. Immunol. 120:121-128.

22. Milner, J.D., Ward, J.M., Keane-Myers, A., and Paul, W.E. 2007. Lymphopenic mice reconstituted with limited repertoire $\mathrm{T}$ cells develop severe, multiorgan, Th2-associated inflammatory disease. Proc. Natl. Acad. Sci. U. S. A. 104:576-581.

23. Jankovic, M., Casellas, R., Yannoutsos, N., Wardemann, H., and Nussenzweig, M.C. 2004. RAGs and regulation of autoantibodies. Annu. Rev. Immunol. 22:485-501.

24. Aalberse, R.C., and Platts-Mills, T.A. 2004. How do we avoid developing allergy: modifications of the $\mathrm{TH} 2$ response from a B-cell perspective. J. Allergy Clin. Immunol. 113:983-986.

\title{
Cutaneous mosaicism: right before our eyes
}

\author{
Jorge Frank ${ }^{1,2}$ and Rudolf Happle ${ }^{3}$
}

1Department of Dermatology and 2Maastricht University Centre for Molecular Dermatology, University Hospital, Maastricht, The Netherlands. ${ }^{3}$ Department of Dermatology, Philipp University of Marburg, Marburg, Germany.

\begin{abstract}
Autosomal recessive cutaneous disorders, including various types of epidermolysis bullosa (EB), usually manifest shortly after birth. The clinical course of these diseases is often characterized by severe complications, limited therapeutic options, and a poor prognosis. A study by Pasmooij et al. reported in this issue of the JCI unravels the molecular mechanisms by which germline mutations in non-Herlitz junctional EB can be corrected in vivo by multiple spontaneously occurring somatic mutational events, a phenomenon known as revertant mosaicism (see the related article beginning on page 1240). These insights open new avenues of thinking for the design of future gene therapy strategies for skin diseases.
\end{abstract}

"I see a bright future for genetics in dermatology and for dermatology in genetics..." - this was the vision of Victor A. McKusick, widely regarded as one of the founding fathers of medical genetics, some 35 years ago (1). Today, it is justified to say that he was absolutely right. During the past 20 years, the genetic basis of more than 350 monogenic skin diseases has been elucidated (2), and the number of disorders awaiting discovery of the underlying gene defect is steadily increasing. However, research in the field of inherited cutaneous disorders is by no means limited to finding novel genes and mutations. Because the skin is easily accessible and, for the trained observer, everything lies "right before your eyes," genetic skin diseases offer unique oppor-

Nonstandard abbreviations used: EB, epidermolysis bullosa; LAMB3, laminin $\beta 3$; LOH, loss of heterozygosity.

Conflict of interest: The authors have declared that no conflict of interest exists.

Citation for this article: J. Clin. Invest. 117:1216-1219 (2007). doi:10.1172/JCI32111. tunities to study complex clinical expression patterns and uncommon inheritance modes and the ability to explore molecular therapeutic regimens such as enzyme replacement strategies and gene therapy $(2,3)$. This notion holds particularly true for cutaneous disorders that clinically present with mosaicism.

\section{Mosaicism}

The term mosaicism refers to the occurrence in an individual of two or more cell populations that are karyotypically or genotypically different and yet are derived from a single zygote (4). Mosaicism can result from a mutation during development that is propagated in only a limited number of the adult cells. In general, any type of cell may be affected by such a mutational event, including gametes (egg and sperm cells), blood cells, and skin cells. The best-known example of mosaicism is mammalian females (normal karyotype 46,XX), which are functional mosaics because one of their X chromosomes is randomly inactivated during embryogenesis (5).
In autosomal recessive mendelian disorders, the underlying mutations often cause embryonic or early postnatal death. Notwithstanding, studying the effects of such mutations at later developmental stages may be possible in individuals carrying the mutation in a mosaic form because gain or loss of genetic functions might be limited to specific cells and tissues or to selected stages of development, as recently shown in various organisms, including, for example, Caenorbabditis elegans, Drosophila, mice, and zebrafish (6-9). Therefore, mosaic organisms are perfectly suited for the investigation of the molecular processes orchestrating very early developmental phases. Further, they may be of use to study a specific cell type or tissue in which a given gene is required to assure proper functioning of signaling pathways and metabolic processes. With respect to organ systems, mosaic organisms can serve to determine whether a particular gene is cell autonomous, i.e., whether the gene is exerting its action exclusively within the cell in which it is expressed or if it also affects neighboring cells that do not manifest a phenotype themselves when carrying a mutation in that gene (8).

\section{Mosaicism in monogenic skin disorders}

Mosaic skin diseases may show different patterns of clinical involvement such as lines of Blaschko, a checkerboard pattern, a phylloid pattern, a patchy pattern without midline separation, and a lateralization 